\title{
Environmental surface sampling for qualitative \& quantitative detection of microbial burden in high risk areas of three hospitals in Dhaka city
}

\author{
Adeeba Khanduker'1, K.M. Shahidul Islam², Lovely Barai², Sk. Shehab Hasan ${ }^{3}$, Mufradul Islam Rafi², \\ Md. Robiul Islam Rubel ${ }^{2}$
}

${ }^{1}$ Department of Microbiology, Labaid Cancer Hospital \& Super Speciality Center, Dhaka, Bangladesh, ${ }^{2}$ Department of Microbiology, BIRDEM General Hospital, Dhaka, Bangladesh, ${ }^{3}$ Department of Microbiology \& Clinical Pathology, National Heart Foundation Hospital \& Research Institute, Dhaka, Bangladesh.

\begin{abstract}
Background and objectives: Acquiring infection from intensive care units \& operation theatres is considered as a significant public health problem around the world. Microbial burden may vary across different ICUs and OTs, albeit these sectors remain underexplored especially in resource poor country. Purpose of the study was qualitative and quantitative assessment of microbial burden in ICUs and OTs' environmental surfaces of three tertiary care hospitals in Dhaka city.
\end{abstract}

Materials and methods: The study was conducted in the department of Microbiology, BIRDEM General Hospital. A total 232 environmental surface swab samples \& 86 hand \& nasal swab samples of ICU staffs were collected from the selected three hospitals. The samples were processed according to standard operating procedures.

Results: Highest bacterial load from inanimate surface was found on sinks $\left(155 \mathrm{CFU} / \mathrm{cm}^{2}\right)$, ICU bedrails $(7.56 \pm 0.76$ $\mathrm{CFU} / \mathrm{cm}^{2}$ ) and OT sandals $\left(66 \mathrm{CFU} / \mathrm{cm}^{2}\right)$. Pathogenic isolates were found more in ICUs \& non-pathogenic isolates were found more in OTs. Among the non-pathogens, Micrococcus spp. (37.8\%) and Bacillus spp. (19.8\%) were found from OTs. Among the pathogens, Acinetobacter spp. was predominant (21.2\%) followed by Pseudomonas spp. (19.2\%) in the ICUs. Overall cleanliness \& hygiene status of Hospital 'A' \& 'C' were found unacceptable whereas Hospital 'B' was found marginally bearable/tolerable.

Conclusion: It could be inferred from the study that health care workers and environmental surface contaminations with MDR pathogens are a definitive risk factor for the indoor patients. Routine sampling to assess bioburden in high risk areas of hospital should be done regularly for identification \& correction of the weakness of ongoing infection control protocol.

\section{Background}

Monitoring of the hospital environment is an essential element in the control of healthcare associated infections (HCAIs) as it contains diverse population of microorganisms and act as highest dissemination reservoir of pathogenic microbes ${ }^{1}$. Among the whole hospital environment, Operating rooms and Intensive care units are regarded as the high risk areas for acquiring HCAIs as intimate handling and care of critically ill patients are given there.

Correspondence:

Adeeba Khanduker

Department of Microbiology

Labaid Cancer Hospital \& Super Speciality Center

Dhaka, Bangladesh.

E-mail: adeebakhanduker@gmail.com
The risk of HCAIs has been estimated to be two to twenty times higher in the developing countries than that of resource-rich countries with the percentage of infected patients exceeding $25 \%$ 2,3,4,5. The rate of HCAIs in Bangladesh may exceed 30\% in some hospitals ${ }^{6}$.

Till the date, only one study conducted in New York University School of Medicine explored the entire microbiome of an Operating Room (OR) by using molecular techniques performed by next generation sequencing of the microbial communities present in three OR environments (found in two different hospitals), and proved that the OR dust contained a microbial community similar to 
the one found on human skin (dominated by Staphylococcus and Corynebacterium) $)^{7}$. Up to $30 \%$ of all surgical site infections (SSIs) are known to be caused by Staphylococcus aureus, especially the methicillin-resistant strains ${ }^{8}$. Besides Staphylococcus spp., other microorganisms which were isolated from ORs are as follows: Enterobacter spp., Micrococcus spp., Acinetobacter spp., Brevibacterium spp., Pseudomonas spp., Klebsiella spp., Bacillus spp. and Escherichia coli $^{9-13}$.

It was found that, among the hospital-acquired infections that develop within the intensive therapy unit, 40-60\% were due to endogenous flora, $20-40 \%$ were due to the contaminated hands of healthcare workers (HCWs), $20-25 \%$ were due to antibiotic-driven change and $20 \%$ were potentially due to environmental contamination ${ }^{14}$. Due to the extreme selective pressure that confinement and cleaning practices induce, microorganisms living in ICUs develop or acquire resistance mechanisms that allow them to survive in the presence of a vast range of antimicrobial agents used in cleaning and antibiotic treatment, to adapt to extremely low nutrient content and to persist on dry surfaces for a long time ${ }^{15}$.

In a study on Microbiology of HCAIs in tertiary hospitals in Dhaka, Bangladesh, the majority of infections were caused by Escherichia coli followed by Pseudomonas, Proteus species, Staphylococcus aureus, Klebsiella species, and Acinetobacter ${ }^{16}$. All infections were found antibiotic resistant which is thought to be due to the widespread use of antibiotics.

The hand contact surfaces, floors, water and air of the hospital environments are the main source of different pathogens that can cause HCAIs ${ }^{14,17}$. Transmission of potential pathogens inside the hospital is complex and involves contaminated hands of healthcare workers, contaminated equipment or contamination of the room from a prior patient. Pathogenic microorganisms persist in the hospital environment from hours to months, which depends on factors such as number, location, biofilm formation, intrinsic resistance of organisms to various cleaning products as well as local conditions. This has been well documented for Staphylococcus aureus, Vancomycinresistant Enterococcus (VRE), Clostridium difficile, Pseudomonas aeruginosa, Acinetobacter $\mathrm{spp}^{18}$.

The main factors associated with the ability of a nosocomial pathogen to survive on inanimate surfaces and equipment are the specific microorganism characteristics (such as genus, species, specific strain, ability to form biofilm and microorganism concentration) and the environmental factors (such as UV radiation, temperature, humidity, presence of organic materials and surface type $)^{19-21}$.

Microbes have an immanent ability to colonize any surface. Studies have shown that microbes persist for weeks on stainless steel surfaces and polymeric materials which are used to fabricate touch surfaces in hospitals ${ }^{22}$. Frequently touched surfaces such as doorknobs, push plates, bed rails, faucet handles and poles supporting intravenous fluid supplies (IV poles) have been identified as reservoirs for the transmission of pathogenic microbes $^{23,24}$. This can easily contaminate hands and equipment of health care workers, who, in turn, can transmit these pathogens to patients during routine care. This proffer is a call for bacteriological standards with which to assess clinical surface hygiene in hospitals, based on those used by the food industry. The first standard concerns any finding of a specific 'indicator' organism, presence of which suggests a requirement for increased cleaning. Indicators include Staphylococcus aureus, including methicillin-resistant Staphylococcus aureus, Clostridium difficile, Vancomycin-resistant Enterococci and various gram-negative bacilli. The second standard concerns about quantitative aerobic colony count of $5 \mathrm{CFU} / \mathrm{cm}^{2}$ on frequent hand touch surfaces in hospitals. The finding of $\geq 5 \mathrm{CFU} / \mathrm{cm}^{2}$ from hand contact surface, whatever the identity of the organisms, indicates that there might be an increased risk of infection for the patient in that environment ${ }^{25}$. This study was conducted for quantitative and qualitative assessment of microbial burden on hospital environmental surfaces and their antimicrobial susceptibility pattern in high risk areas (Operation theatres and Intensive care units) of three hospitals of Dhaka city.

\section{Materials And Methods}

Study Setting, Design, and Period. A hospital-based cross-sectional observational study was conducted from 15 March 2019 to 30 February 2020 at Department of Microbiology, BIRDEM General Hospital, Dhaka. Three hospitals were included in the present study which were selected by purposive sampling. Those hospitals were designated as hospital A, hospital B and hospital C in order to maintain the confidentiality of the hospitals. Hospital A is a private general hospital. It is a multidisciplinary hospital complex consisting of 103 cabins, 747 ward beds, 21 beds in ICU and 8 Operation theatres in the 
OT complex. Hospital B is a mono disciplinary private specialized hospital for cardiac treatment. It consists of 78 beds for in patient management including 8 beds in ICU and 2 Operation theatres. Hospital $\mathrm{C}$ is a tertiary level government general hospital consisting of around 1700 beds, 36 ICU beds in 3 ICUs and 12 Operation theatres in the main OT complex. The samples were collected in the following manner.

\section{Operation theatre}

In Hospital 'A' 5 OT were selected out of 8 OT.

In Hospital 'B' samples were taken from 2 OT as it consists 2 OT.

In Hospital 'C' 5 OT were selected randomly as every second or third out of 12 OT in the main OT complex in order to keep the sample size same with Hospital 'A'.

So, in total samples were taken from $12(5+2+5)$ OTs of three hospitals. From these 12 OTs, samples were taken from floor, walls, door handle, OT light, IV pole, OT table, sucker, anesthesia machine and diathermy machine. OT sandal, instrument trolley, a sterile instrument, fine scissor, sterile OT gown, sterile draping sheet and sterile kidney tray were collected as single sample from each hospital.

As OT floor, light source and table were routinely cleaned, here the samples were taken half an hour before and after routine cleaning.

\section{Intensive Care Unit}

Sites for sample collection were chosen as frequently or high touch surfaces, such as bedrail, over bed table, IV pole and stethoscope. As Hospital 'A' and 'C' ICU were 21 and 20 bedded respectively so samples were taken from every $3^{\text {rd }}$ bed to cover the whole ICU.

Along with the above mentioned 4 site samples were also taken from wall, floor and sink. As the floor was only routinely cleaned, here the samples were taken half an hour before and after routine cleaning. Hand and nasal swabs were also taken from the on duty ICU staffs to see the rate of colonization.

Bacteriological Sampling and Culture. A total 318 bacteriological samples were collected for analysis from three tertiary care hospitals. Among those 232 were inanimate surface samples and 86 human samples from ICU staffs only. Out of 232 inanimate surface samples 91 , $50 \& 91$ samples each were taken from Hospital 'A', 'B' and ' $C$ ' respectively. Among the 86 human samples 43 were hand swabs and 43 were nasal swabs. Sixteen hand swabs and 16 nasal swabs were taken from Hospital 'A', 12 hand and 12 nasal swabs were taken from Hospital ' $\mathrm{B}$ ' and 15 hand and 15 nasal swabs were taken from Hospital 'C'.

Inanimate surfaces to be sampled were screened by sterile cotton swabs by two methods: (I) Non-enrichment method (II) Enrichment method.

\section{Collection of hand and nasal swabs}

For hand and nasal swab of ICU staffs, sterile cotton swabs were moistened in $5 \mathrm{ml}$ of $0.9 \%$ sterile normal saline solution and rubbed over the hand (specially the finger webs and nails) and anterior nares. Then brought to the laboratory and inoculated in Blood \& MacConkey agar media and incubated overnight into an incubator at $37^{\circ} \mathrm{C}$. The isolated organisms from different samples were identified by standard microbiological techniques such as colony morphology, microscopic features and standard phenotypic characters.

Antimicrobial Susceptibility Testing. Antimicrobial susceptibility profile of the isolates was performed based on the Kirby-Bauer agar disc diffusion method. The suspension of the identified test organism was prepared from similar colonies. The densities of suspension were determined by comparing with McFarland 0.5 Barium sulfate solutions ${ }^{26}$.

Data Analysis. All data were entered, cleaned, and analyzed using Statistical Software Package for Social Sciences (SPSS) version 23 (SPSS Inc., Chicago, IL, USA) for Windows. Generated data were compiled and presented using descriptive statistics.

\section{Results}

Table 3.1 shows the distribution of surface samples according to the collection sites in ICUs and OTs respectively. From the total 232 inanimate surface samples 87 were collected from the three ICUs and 145 were collected from the three OTs. 
Table 3.1: Distribution of inanimate surface samples taken from three ICUs and three OTs according to collection site.

\begin{tabular}{|c|c|c|c|c|}
\hline \multirow{2}{*}{ Sample collection site } & & \multicolumn{3}{|c|}{ Frequency of samples No. (\%) } \\
\hline & & Hospital 'A' & Hospital 'B' & Hospital ' $C$ ' \\
\hline \multicolumn{5}{|l|}{ ICU samples } \\
\hline Bedrail & $(\mathrm{n}=21)$ & $8(38.1)$ & $5(23.8)$ & $8(38.1)$ \\
\hline Over bed table & $(\mathrm{n}=19)$ & $7(36.8)$ & $5(26.3)$ & $7(36.8)$ \\
\hline IV pole & $(\mathrm{n}=18)$ & $7(38.9)$ & $4(22.2)$ & $7(38.9)$ \\
\hline Stethoscope & $(n=14)$ & $5(35.7)$ & $4(28.6)$ & $5(35.7)$ \\
\hline Floor & $(\mathrm{n}=8)$ & $3(37.5)$ & $2(25.0)$ & $3(37.5)$ \\
\hline Wall & $(\mathrm{n}=4)$ & $1(25.0)$ & $2(50.0)$ & $1(25.0)$ \\
\hline Sink & $(\mathrm{n}=3)$ & $1(33.33)$ & $1(33.33)$ & $1(33.33)$ \\
\hline Total & $(\mathbf{N}=87)$ & $31(35.6)$ & $25(28.7)$ & $31(35.6)$ \\
\hline \multicolumn{5}{|l|}{ OT samples } \\
\hline Wall & $(\mathrm{n}=12)$ & $5(41.7)$ & $2(16.6)$ & $5(41.7)$ \\
\hline Floor & $(\mathrm{n}=24)$ & $10(41.7)$ & $4(16.6)$ & $10(41.7)$ \\
\hline OT light & $(\mathrm{n}=12)$ & $5(41.7)$ & $2(16.6)$ & $5(41.7)$ \\
\hline IV pole & $(\mathrm{n}=12)$ & $5(41.7)$ & $2(16.6)$ & $5(41.7)$ \\
\hline OT table & $(\mathrm{n}=12)$ & $5(41.7)$ & $2(16.6)$ & $5(41.7)$ \\
\hline Door handle & $(\mathrm{n}=12)$ & $5(41.7)$ & $2(16.6)$ & $5(41.7)$ \\
\hline Sucker & $(\mathrm{n}=12)$ & $5(41.7)$ & $2(16.6)$ & $5(41.7)$ \\
\hline Anaesthesia machine & $e(n=12)$ & $5(41.7)$ & $2(16.6)$ & $5(41.7)$ \\
\hline Diathermy machine & $(\mathrm{n}=12)$ & $5(41.7)$ & $2(16.6)$ & $5(41.7)$ \\
\hline Sink & $(\mathrm{n}=3)$ & $1(33.33)$ & $1(33.33)$ & $1(33.33)$ \\
\hline OT sandal & $(n=3)$ & $1(33.33)$ & $1(33.33)$ & $1(33.33)$ \\
\hline Instrument trolley & $(\mathrm{n}=3)$ & $1(33.33)$ & $1(33.33)$ & $1(33.33)$ \\
\hline Sterile instrument & $(\mathrm{n}=3)$ & $1(33.33)$ & $1(33.33)$ & $1(33.33)$ \\
\hline Fine scissor & $(\mathrm{n}=2)$ & $1(50.0)$ & Nil & $1(50.0)$ \\
\hline OT gown & $(\mathrm{n}=3)$ & $1(33.33)$ & $1(33.33)$ & $1(33.33)$ \\
\hline Sterile draping sheet & $\mathrm{t}(\mathrm{n}=3)$ & $1(33.33)$ & $1(33.33)$ & $1(33.33)$ \\
\hline Sterile kidney tray & $(\mathrm{n}=3)$ & $1(33.33)$ & $1(33.33)$ & $1(33.33)$ \\
\hline \multicolumn{2}{|c|}{$0.2 \%$ Glutaraldehyde $(\mathrm{n}=2)$} & $1(50.0)$ & Nil & $1(50.0)$ \\
\hline Total $(\mathrm{N}$ & $\mathrm{N}=145)$ & $59(40.7)$ & 27 (18.6) & $59(40.7)$ \\
\hline
\end{tabular}

Table 3.2 shows the mean $( \pm$ SD) bacterial load from the inanimate surface samples in the three ICUs and OTs. Among the ICUs, the highest bacterial load was found on the sink of Hospital 'A' $\left(155.00 \pm 0.00 \mathrm{CFU} / \mathrm{cm}^{2}\right)$ and the lowest load was on the stethoscope of Hospital 'B' (1.10 $\left.\pm 0.26 \mathrm{CFU} / \mathrm{cm}^{2}\right)$. Among the OTs, highest load was found on the OT sandal of Hospital 'A' (68.00 \pm 0.00 $\mathrm{CFU} / \mathrm{cm}^{2}$ ) and the lowest load was found on the IV pole of Hospital 'B' $\left(0.70 \pm 0.28 \mathrm{CFU} / \mathrm{cm}^{2}\right)$. No growth was found on the sterilized equipment in all the OTs.
Table 3.2: Mean bacterial load \& hygiene status of inanimate surface samples in three ICUs and OTs.

\begin{tabular}{|c|c|c|c|c|}
\hline \multirow{2}{*}{ Sample sites } & \multicolumn{3}{|c|}{$\begin{array}{c}\text { Mean }( \pm \mathrm{SD}) \text { of Bacterial load in CFU/cm2(Hygiene } \\
\text { status) }\end{array}$} & \multirow{2}{*}{$\begin{array}{c}\text { ANOVA } \\
\text { test } \\
\text { p-value }\end{array}$} \\
\hline & Hospital 'A' & Hospital 'B' & Hospital 'C' & \\
\hline \multicolumn{5}{|l|}{ ICU samples } \\
\hline Bed rail & $6.40 \pm 0.97(\mathrm{UA})$ & $4.72 \pm 0.37(\mathrm{~A})$ & $7.56 \pm 0.76(\mathrm{UA})$ & $\begin{array}{l}A \text { vs } B=0.005 \\
A \text { vs } C=0.027 \\
B \text { vs } C=<0.001\end{array}$ \\
\hline Over bed table & $6.62 \pm 0.53(\mathrm{UA})$ & $4.94 \pm 0.57(\mathrm{~A})$ & $5.84 \pm 2.22(\mathrm{UA})$ & A vs $B=0.182$ \\
\hline IV pole & $6.06 \pm 0.46(\mathrm{UA})$ & $3.00 \pm 0.63(\mathrm{~A})$ & $6.21 \pm 0.39(\mathrm{UA})$ & $\begin{array}{l}\text { A vs } B=<0.001 \\
B \text { vs } C=<0.001\end{array}$ \\
\hline Stethoscope & $2.84 \pm 0.30(\mathrm{~A})$ & $1.10 \pm 0.26(\mathrm{~A})$ & $2.12 \pm 1.15(\mathrm{~A})$ & $A$ vs $B=0.013$ \\
\hline Wall * & 5.40 (UA) & $3.80(\mathrm{~A})$ & 5.90 (UA) & Not done \\
\hline Sink * & 155.00 (UA) & $110.00(\mathrm{UA})$ & $120.00(\mathrm{UA})$ & Not done \\
\hline \multicolumn{5}{|l|}{ OT samples } \\
\hline Wall & $4.16 \pm 0.48(\mathrm{~A})$ & $4.50 \pm 0.42(\mathrm{~A})$ & $6.30 \pm 0.59$ (UA) & $\begin{aligned} \text { A vs } C & =<0.001 \\
B \text { vs } C & =0.008\end{aligned}$ \\
\hline IV pole & $4.92 \pm 0.99(\mathrm{~A})$ & $0.70 \pm 0.28(\mathrm{~A})$ & $6.50 \pm 1.41(\mathrm{UA})$ & $\begin{array}{l}A \text { vs } B=0.005 \\
B \text { vs } C=0.001\end{array}$ \\
\hline Door handle & $4.92 \pm 0.99(\mathrm{~A})$ & $3.50 \pm 0.71(\mathrm{~A})$ & $7.64 \pm 0.95(\mathrm{UA})$ & $\begin{array}{l}\mathrm{A} \text { vs } \mathrm{C}=0.004 \\
\mathrm{~B} \text { vs } \mathrm{C}=0.002\end{array}$ \\
\hline Sucker & $5.40 \pm 0.94(\mathrm{UA})$ & $0.00(\mathrm{~A})$ & $16.40 \pm 12.79(\mathrm{UA})$ & $\mathrm{B}$ vs $\mathrm{C}=0.143$ \\
\hline Anaesthesia machine & $10.28 \pm 14.45$ (UA) & $2.00 \pm 0.57(\mathrm{~A})$ & $7.18 \pm 0.72(\mathrm{UA})$ & A vs $B=0.995$ \\
\hline Diathermy machine & $6.64 \pm 0.74(\mathrm{UA})$ & $4.85 \pm 1.06(\mathrm{~A})$ & $7.54 \pm 1.06(\mathrm{UA})$ & A vs $B=0.995$ \\
\hline Sink * & 48.00 (UA) & 33.10 (UA) & 66.00 (UA) & Not done \\
\hline OT Sandal * & 68.00 (UA) & 48.00 (UA) & 60.00 (UA) & Not done \\
\hline Instrument trolley * & $3.20(\mathrm{~A})$ & $0.00(\mathrm{~A})$ & $5.80(\mathrm{UA})$ & Not done \\
\hline Sterile instrument * & 0 & 0 & 0 & Not done \\
\hline Fine scissors * & 0 & - & 0 & Not done \\
\hline OT gown * & 0 & 0 & 0 & Not done \\
\hline Sterile draping sheet * & 0 & 0 & 0 & Not done \\
\hline Sterile kidney tray * & 0 & 0 & 0 & Not done \\
\hline $0.2 \%$ Glutaraldehyde * & 0 & - & 0 & Not done \\
\hline
\end{tabular}

- Acceptable bacterial load on inanimate surface sample is $<5 \mathrm{CFU} / \mathrm{cm}^{2}$ [28]

- $\mathrm{UA}=$ Unacceptable, $\mathrm{A}=$ Acceptable

* Statistical analysis of bacterial load were not done due to single sample size from each hospital.

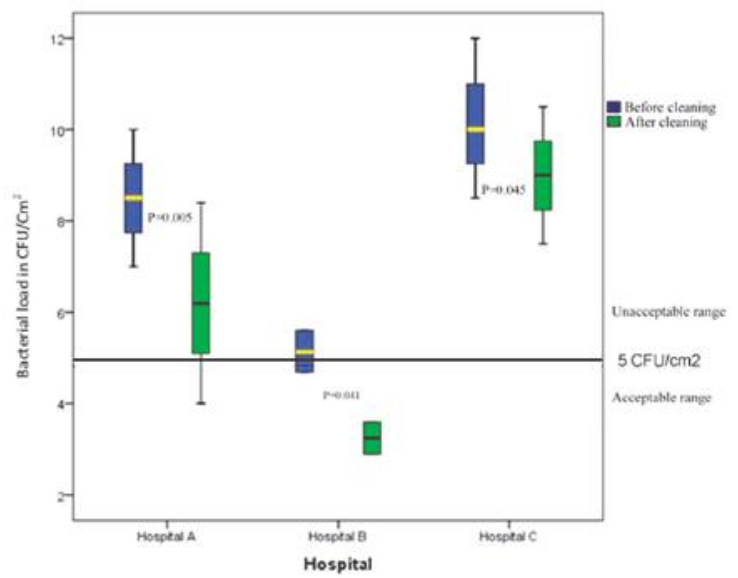

Figure 3.1: Box and Whisker plot shows the observation of bacterial load on ICU floor before and after routine cleaning. In all three hospitals, reduction of bacterial load was found statistically significant $(p<0.05)$ but the burden remained in unacceptable range $(>5$ CFU/cm²) in Hospital ' $A$ ' \& ' $C$ '. 


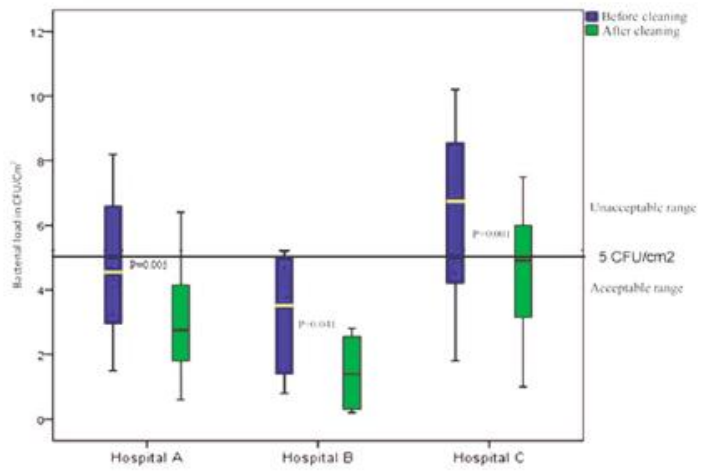

Figure 3.2: Box and Whisker plot shows the observation of bacterial load on inanimate surfaces (floor, light and table) before and after routine cleaning. Here reduction of bacterial load was found statistically significant $(p<0.05)$ and in acceptable range $(<5$ $\left.\mathrm{CFU} / \mathrm{cm}^{2}\right)$ in all three hospitals.
Out of 87 ICU inanimate surfaces, growth was found in $74.7 \%$ (65) samples. Highest growth positive inanimate surfaces were floor, wall \& sink (100\%). And out of 145 OT inanimate surfaces, growth was found in $70.3 \%$ (102) samples. Highest growth positive inanimate surfaces were OT sandal \& sink (100\%). Micrococcus spp. was found highest among the ICUs and OTs. Details of specimen and bacterial isolates are depicted in Table $3.3 \& 3.4$. Mixed bacterial flora were isolated from various sites. No organisms were found on sterile instruments, fine scissor, sterile kidney tray, sterile OT gown, draping sheet and from in use $0.2 \%$ Glutaraldehyde. Isolation rate of gram negative organism was significantly higher in ICU of Hospital 'A' \& 'C', whereas frequency of gram positive bacteria was significantly more in OTs of Hospital 'A' (p $<0.001)$ \& Hospital 'C' $(\mathrm{p}<0.001)$ (Table 3.5). Potentially pathogenic organisms were found significantly more $(\mathrm{p}$ $<0.001$ ) in Hospital 'A' \& 'C' ICU, where in all OTs non-pathogenic organisms were found more (Table 3.6)

Table 3.3: Frequency of organisms isolated from different collection sites of ICU environments in three hospitals

\begin{tabular}{|c|c|c|c|c|c|c|c|c|c|c|c|c|c|}
\hline \multirow[b]{2}{*}{$\begin{array}{l}\text { Sampling } \\
\text { Sites }\end{array}$} & \multirow{2}{*}{$\begin{array}{l}\text { No. of } \\
\text { isolates } \\
\text { (n) }\end{array}$} & \multicolumn{6}{|c|}{ No. $(\%)$ of gram positive isolates } & \multicolumn{6}{|c|}{ No. $(\%)$ of gram negative isolates } \\
\hline & & MSSA & MRSA & CoNS & $\begin{array}{l}\text { Bacillus } \\
\text { spp. }\end{array}$ & $\begin{array}{l}\text { Microco } \\
\text { ccus } \\
\text { spp. }\end{array}$ & $\begin{array}{l}\text { Gr. D } \\
\text { Entero } \\
\text { coccus }\end{array}$ & E. coli & $\begin{array}{l}\text { Klebsiella } \\
\text { spp.. }\end{array}$ & $\begin{array}{l}\text { Entero } \\
\text { bacter } \\
\text { spp. }\end{array}$ & $\begin{array}{l}\text { Pseudo } \\
\text { monas } \\
\text { spp. }\end{array}$ & $\begin{array}{l}\text { Flavob } \\
\text { acterium } \\
\text { spp. }\end{array}$ & $\begin{array}{l}\text { Acinetob } \\
\text { acter } \\
\text { spp. }\end{array}$ \\
\hline \multicolumn{14}{|c|}{ Inanimate surfaces } \\
\hline Bedrail & 46 & $1(2.2)$ & $2(4.3)$ & $2(4.3)$ & $5(10.9)$ & $6(13.04)$ & $1(2.2)$ & $2(4.3)$ & $4(8.7)$ & $3(6.52)$ & $9(19.6)$ & $2(4.3)$ & $9(19.6)$ \\
\hline $\begin{array}{l}\text { Over bed } \\
\text { table }\end{array}$ & 34 & 0 & 0 & 0 & $4(11.8)$ & $7(20.6)$ & 0 & 0 & $5(14.7)$ & 0 & $10(29.4)$ & 0 & $8(23.5)$ \\
\hline IV pole & 21 & $1(4.76)$ & 0 & 0 & $2(9.5)$ & $5(23.8)$ & 0 & $2(9.5)$ & $2(9.5)$ & 0 & $2(9.5)$ & $1(4.76)$ & $6(28.6)$ \\
\hline Stethoscope & 15 & $1(6.67)$ & 0 & 0 & $2(13.3)$ & $4(26.7)$ & 0 & $1(6.67)$ & 0 & 0 & $2(13.3)$ & 0 & $5(23.8)$ \\
\hline Floor & 16 & $1(6.25)$ & $1(6.25)$ & 0 & $1(6.25)$ & $5(31.25)$ & 0 & 0 & 0 & 0 & $2(12.5)$ & $1(6.25)$ & $5(31.25)$ \\
\hline Wall & 13 & 0 & 0 & 0 & $3(23.1)$ & $3(23.1)$ & 0 & 0 & 0 & 0 & $2(15.4)$ & $5(38.5)$ & 0 \\
\hline Sink & 11 & 0 & 0 & 0 & $1(9.1)$ & $4(36.4)$ & 0 & $2(18.2)$ & $1(9.1)$ & 0 & $3(27.8)$ & 0 & 0 \\
\hline Total & $\mathrm{N}=156$ & $4(2.56)$ & $3(1.92)$ & $2(1.28)$ & 18 (11.5) & $34(21.8)$ & $1(0.6)$ & 7 (4.49) & $12(7.69)$ & $3(1.92)$ & $30(19.2)$ & $9(5.76)$ & $33(21.2)$ \\
\hline
\end{tabular}

- CoNS = Coagulase negative Staphylococcus, MRSA = Methicillin resistant Staphylococcus aureus, MSSA = Methicillin sensitive Staphylococcus aureus 
Table 3.4: Frequency of organisms isolated from different collection sites of OT environments in three hospitals

\begin{tabular}{|c|c|c|c|c|c|c|c|c|c|c|c|c|c|}
\hline \multirow[b]{2}{*}{ Sampling Sites } & \multirow{2}{*}{$\begin{array}{l}\text { No. of } \\
\text { isolates } \\
\text { (n) }\end{array}$} & \multicolumn{6}{|c|}{ No. (\%) of gram positive isolates } & \multicolumn{6}{|c|}{ No. $(\%)$ of gram negative isolates } \\
\hline & & MSSA & MRSA & CoNS & $\begin{array}{l}\text { Bacillus } \\
\text { spp. }\end{array}$ & $\begin{array}{l}\text { Microco } \\
\text { ccus } \\
\text { spp. }\end{array}$ & $\begin{array}{l}\text { Gr. D } \\
\text { Entero } \\
\text { coccus } \\
\end{array}$ & E.coli & $\begin{array}{l}\text { Klebsie } \\
\text { lla spp. }\end{array}$ & $\begin{array}{l}\text { Entero } \\
\text { bacter } \\
\text { spp. }\end{array}$ & $\begin{array}{l}\text { Pseudo } \\
\text { monas } \\
\text { spp. }\end{array}$ & $\begin{array}{l}\text { Flavobac } \\
\text { terium } \\
\text { spp. }\end{array}$ & $\begin{array}{l}\text { Acinet } \\
\text { obacter } \\
\text { spp. }\end{array}$ \\
\hline \multicolumn{14}{|l|}{ Inanimate surface } \\
\hline Wall & 21 & $2(9.5)$ & 0 & $1(4.76)$ & $7(33.33)$ & $8(38.1)$ & 0 & 0 & 0 & 0 & $1(4.76)$ & $2(9.5)$ & 0 \\
\hline Floor & 26 & 0 & 0 & $1(3.85)$ & $5(19.23)$ & $6(23.1)$ & 0 & $2(7.7)$ & 0 & $5(19.2)$ & $1(3.85)$ & $2(7.7)$ & $4(15.4)$ \\
\hline IV stand & 17 & 0 & 0 & 0 & $3(17.6)$ & $10(58.8)$ & 0 & 0 & 0 & 0 & 0 & $1(5.9)$ & $3(17.6)$ \\
\hline OT table & 14 & $2(14.3)$ & 0 & 0 & $3(21.4)$ & $7(50.0)$ & 0 & 0 & 0 & 0 & $1(7.14)$ & $1(7.14)$ & 0 \\
\hline Door handle & 22 & $3(13.6)$ & 0 & 0 & $5(22.7)$ & $9(40.9)$ & 0 & 0 & 0 & 0 & $1(4.54)$ & $4(18.2)$ & 0 \\
\hline Sucker & 21 & 0 & 0 & 0 & $3(14.3)$ & $6(28.6)$ & $2(9.5)$ & 0 & 0 & 0 & $2(9.5)$ & $5(23.8)$ & $3(14.3)$ \\
\hline $\begin{array}{l}\text { Anaesthesia } \\
\text { machine }\end{array}$ & 18 & 0 & 0 & 0 & $5(27.8)$ & $8(44.4)$ & $2(11.1)$ & 0 & 0 & 0 & 0 & $3(16.6)$ & 0 \\
\hline $\begin{array}{l}\text { Diathermy } \\
\text { machine }\end{array}$ & 17 & 0 & 0 & 0 & $5(29.4)$ & $8(47.1)$ & 0 & 0 & 0 & 0 & $2(11.7)$ & $2(11.7)$ & 0 \\
\hline Sink & 20 & 0 & 0 & 0 & 0 & $4(20)$ & 0 & $3(15)$ & 0 & 0 & $7(35)$ & $6(30)$ & 0 \\
\hline OT sandal & 31 & $1(3.22)$ & 0 & $2(6.4)$ & $5(16.1)$ & $8(25.8)$ & 0 & $1(3.22)$ & $4(12.9)$ & $4(12.9)$ & $3(9.7)$ & $3(9.7)$ & 0 \\
\hline Instrument trolley & 10 & 0 & 0 & 0 & $2(20)$ & $8(80)$ & 0 & 0 & 0 & 0 & 0 & 0 & 0 \\
\hline Total & 217 & $8(3.68)$ & $\mathbf{0}$ & $4(1.84)$ & $43(19.8)$ & $82(37.8)$ & $4(1.84)$ & $6(2.76)$ & $4(1.84)$ & $9(4.14)$ & $18(8.3)$ & $29(13.4)$ & $10(4.6)$ \\
\hline
\end{tabular}

- CoNS = Coagulase negative Staphylococcus, MRSA = Methicillin resistant S. aureus, MSSA = Methicillin sensitive S. aureus

- Sterile instruments, draping sheet, kidney tray, OT gown, $0.2 \%$ Glutaraldehyde were not shown separately due to spaces \& culture revealed no growth.

Table 3.5: Rate of isolation of gram reactive organisms in ICUs \& OTs of different hospitals

\begin{tabular}{cccc}
\hline High risk area & \multicolumn{2}{c}{ Rate of isolation No. (\%) } & $\begin{array}{c}\text { Chi-square } \\
\text { test } \\
\text { p-value }\end{array}$ \\
\cline { 2 - 3 } & gram negative & gram positive & \\
\hline Hospital 'A' & & $19(34.6)$ & $<0.001$ \\
ICU (n = 55) & $36(65.4)$ & $48(71.7)$ & $<0.001$ \\
OT (n=67) & $19(28.3)$ & $30(62.5)$ & 0.920 \\
Hospital 'B' & & $30(68.2)$ & 0.632 \\
ICU (n=48) & $18(37.5)$ & & \\
OT (n=44) & $14(31.8)$ & $13(24.5)$ & 0.001 \\
Hospital 'C' & & $63(59.4)$ & 0.001 \\
ICU (n=5) & $40(75.5)$ & & \\
OT (n=106) & $43(40.6)$ & &
\end{tabular}

Table 3.6: Frequency of pathogenic \& non-pathogenic bacteria in three ICUs \& OTs of three different hospitals

\begin{tabular}{cccc}
\hline High risk area & \multicolumn{2}{c}{ No. (\%) of bacteria } & $\begin{array}{c}\text { Chi-square } \\
\text { test } \\
\text { p-value }\end{array}$ \\
\cline { 2 - 3 } & Potentially pathogenic & Non-pathogenic & \\
\hline Hospital 'A' & $39(69.6)$ & $17(30.4)$ & $<0.001$ \\
ICU (n=56) & $21(30.9)$ & $47(69.1)$ & $<0.001$ \\
OT (n=68) & & & \\
Hospital 'B' & $15(32.6)$ & $31(67.4)$ & 0.231 \\
ICU (n=46) & $15(34.8)$ & $28(65.2)$ & 0.256 \\
OT (n=43) & & & $<0.001$ \\
Hospital 'C' & $48(88.9)$ & $6(11.1)$ & $<0.001$ \\
ICU (n=54) & $44(41.5)$ & $62(58.5)$ & \\
OT (n=106) & & & \\
\hline
\end{tabular}

Frequency of bacterial colonization among the ICU staffs are shown in table 3.7. In hand swabs, $20 \%$ S. aureus was found in Hospital 'A', Enterobacteriaceae was found $20 \%$ in Hospital 'A' and $42.9 \%$ in Hospital 'C', Pseudomonas spp. was found $20 \%$ in Hospital 'A'. MRSA was detected only $11.8 \%$ from nasal swab of Hospital 'C' ICU staffs. 
Table 3.7: Frequency of bacterial colonization in hand \& nose of ICU staffs of three hospitals

\begin{tabular}{ccccc}
\hline \multirow{2}{*}{$\begin{array}{c}\text { Site of } \\
\text { sample }\end{array}$} & Category of bacteria & \multicolumn{3}{c}{ Frequency of bacterial isolates No. (\%) } \\
\cline { 3 - 5 } & Hospital 'A' & Hospital 'B' & Hospital 'C' \\
\hline \multirow{4}{*}{ Hand swab } & S. aureus & $1(20.0)$ & 0 & 0 \\
& CoNS & 0 & 0 & 0 \\
& MRSA & 0 & 0 & 0 \\
& Enterobacteriaceae & $1(20.0)$ & 0 & $3(42.9)$ \\
& Pseudomonas spp. & $1(20.0)$ & 0 & 0 \\
& Non-pathogenic & $2(40.0)$ & $6(100)$ & $4(571)$ \\
\hline \multirow{4}{*}{ Nasal swab } & Total & 5 & 6 & 7 \\
\hline & S. aureus & $2(22.2)$ & $2(25.0)$ & $4(23.5)$ \\
& CoNS & $2(22.2)$ & $2(25.0)$ & $3(17.6)$ \\
& MRSA & 0 & 0 & $2(11.8)$ \\
& Enterobacteriaceae & $2(22.2)$ & 0 & $4(23.5)$ \\
& Pseudomonas spp. & 0 & 0 & $1(5.9)$ \\
& Non-pathogenic & $3(33.3)$ & $4(50.0)$ & $3(17.6)$ \\
\hline Total & 9 & 8 & 17 \\
\hline
\end{tabular}

The antimicrobial drug resistance patterns of isolated Enterobacteriaceae, Acinetobacter spp., Pseudomonas spp. and Staphylococcus spp. from environment of ICUs \& OTs of three hospitals are depicted in Table 3.8. Based on antibiogram of 3rd generation Cephalosporins, Amoxiclav and Aztreonam, approximately 53.3\%, 40.0\% \& 71.4\% were detected as ESBL producers in Hospital 'A', 'B' \& 'C' respectively. Methicillin resistant Staphylococcus aureus (MRSA) were found in ICU of Hospital 'A' $(16.7 \%)$ \& Hospital 'C' $(30.0 \%)$.

Table 3.8: Antimicrobial drug resistance patterns of isolated Enterobacteriaceae, Acinetobacter spp., Pseudomonas spp. and Staphylococcus spp. from environment of ICUs \& OTs of three hospitals

\begin{tabular}{|c|c|c|c|c|c|c|c|c|c|c|c|c|}
\hline \multirow[t]{2}{*}{ Antibiotics tested } & \multicolumn{3}{|c|}{ Enterobacteriaceae } & \multicolumn{3}{|c|}{ Pseudomonas spp. } & \multicolumn{3}{|c|}{ Acinetobacter spp. } & \multicolumn{3}{|c|}{ S. aureus } \\
\hline & $\begin{array}{l}\text { Hos 'A' } \\
n=15\end{array}$ & $\begin{array}{c}\text { Hos 'B' } \\
n=5\end{array}$ & $\begin{array}{l}\text { Hos 'C' } \\
n=21\end{array}$ & $\begin{array}{l}\text { Hos 'A' } \\
n=15\end{array}$ & $\begin{array}{c}\text { Hos 'B' } \\
n=8\end{array}$ & $\begin{array}{l}\text { Hos 'C' } \\
n=25\end{array}$ & $\begin{array}{l}\text { Hos 'A' } \\
\mathrm{n}=18\end{array}$ & $\begin{array}{c}\text { Hos 'B' } \\
n=4\end{array}$ & $\begin{array}{l}\text { Hos 'C' } \\
n=21\end{array}$ & $\begin{array}{c}\text { Hos 'A' } \\
n=6\end{array}$ & $\begin{array}{c}\text { Hos 'B' } \\
n=5\end{array}$ & $\begin{array}{l}\text { Hos 'C' } \\
n=10\end{array}$ \\
\hline Ceftriaxone & $8(53.33)$ & $2(40.0)$ & $15(71.42)$ & - & - & - & $14(77.78)$ & $2(50.0)$ & $16(76.19)$ & - & - & - \\
\hline Ceftazidime & $8(53.33)$ & $2(40.0)$ & $15(71.42)$ & $9(60.0)$ & $4(50.0)$ & $14(56.0)$ & $14(77.78)$ & $2(50.0)$ & $16(76.19)$ & - & - & - \\
\hline Cefotaxime & $8(53.33)$ & $2(40.0)$ & $15(71.42)$ & - & - & - & $14(77.78)$ & $2(50.0)$ & $16(76.19)$ & - & - & - \\
\hline Cefixime & $8(53.33)$ & $2(40.0)$ & $15(71.42)$ & - & - & - & $14(77.78)$ & $2(50.0)$ & $16(76.19)$ & - & - & - \\
\hline Amoxiclav & $8(53.33)$ & $2(40.0)$ & $15(71.42)$ & - & - & - & $14(77.78)$ & $2(50.0)$ & $16(76.19)$ & $2(33.3)$ & $1(20.0)$ & $2(20.0)$ \\
\hline $\begin{array}{l}\text { Piperacillin- } \\
\text { Tazobactum (PIT) }\end{array}$ & $3(20.0)$ & 0 & $6(28.57)$ & $6(40.0)$ & $3(37.5)$ & $12(48.0)$ & $14(77.78)$ & $2(50.0)$ & $15(71.42)$ & - & - & - \\
\hline Aztreonam & $8(53.33)$ & $2(40.0)$ & $15(71.42)$ & $7(46.67)$ & $2(25.0)$ & $13(52.0)$ & $14(77.78)$ & $2(50.0)$ & $16(76.19)$ & - & - & - \\
\hline Imipenem & $1(6.67)$ & 0 & $4(19.05)$ & $7(46.67)$ & $4(50.0)$ & $14(56.0)$ & $14(77.78)$ & $2(50.0)$ & $15(71.42)$ & - & - & - \\
\hline Amikacin & $2(13.33)$ & 0 & $6(28.57)$ & $5(33.33)$ & $2(25.0)$ & $12(48.0)$ & $14(77.78)$ & $2(50.0)$ & $15(71.42)$ & 0 & 0 & $1(10.0)$ \\
\hline Gentamicin & $2(13.33)$ & 0 & $6(28.57)$ & $5(33.33)$ & $2(25.0)$ & $13(52.0)$ & $14(77.78)$ & $2(50.0)$ & $15(71.42)$ & 0 & 0 & $1(10.0)$ \\
\hline Ciprofloxacin & $4(26.67)$ & $1(20.0)$ & $4(19.05)$ & $6(40.0)$ & $3(37.5)$ & $12(48.0)$ & $14(77.78)$ & $2(50.0)$ & $15(71.42)$ & $1(16.67)$ & $1(20.0)$ & $2(20.0)$ \\
\hline Cotrimoxazole & $2(13.33)$ & $1(20.0)$ & $5(23.81)$ & $12(80.0)$ & $6(75.0)$ & $20(80.0)$ & $14(77.78)$ & $2(50.0)$ & $16(76.19)$ & $1(16.67)$ & $1(20.0)$ & $2(20.0)$ \\
\hline Cefoxitin & - & - & - & - & - & - & - & - & - & $1(16.67)$ & 0 & $3(30.0)$ \\
\hline Oxacillin & - & - & - & - & - & - & - & - & - & $1(16.67)$ & 0 & $3(30.0)$ \\
\hline
\end{tabular}




\section{Discussion}

To prevent the consequences and to reduce the microbial burden maintaining environmental hygiene is a must. Monitoring environmental hygiene by the microbiological testing of surfaces and equipment is useful to detect changing trends of types and counts of microbial flora. With this background this study was undertaken to observe environmental hygiene in three tertiary care hospitals of Dhaka city by quantitative and qualitative assessment of microbial burden.

In any hospital setting, air, water, medical devices dry surfaces as well as healthcare workers all act as potential sources of contamination and infection. In this study, sampling of the hospital environment was done from frequently touched inanimate surfaces from ICU and OT; nasal and hand swab samples from ICU staffs were also taken to identify the source of infection and carrier of MDR organism.

From the frequently or high touched inanimate surfaces, swab samples were taken from unit surface area of the three hospitals. The mean bacterial burden on inanimate surfaces of ICUs (Table 3.2) was found $481.35 \mathrm{CFU} / \mathrm{cm}^{2}$ and of OTs was found $442.64 \mathrm{CFU} / \mathrm{cm}^{2}$. Highest burden was observed over the sinks $\left(117-385 \mathrm{CFU} / \mathrm{cm}^{2}\right)$, OT sandals $\left(176 \mathrm{CFU} / \mathrm{cm}^{2}\right)$ and ICU bedrails $\left(18.68 \mathrm{CFU} / \mathrm{cm}^{2}\right)$. No published data regarding colony count from samples of sinks of ICU and OT were available to compare with the findings of the present study. Heavy contamination of bedrails was reported by Shams et $\mathrm{al}^{27}$. and Dancer et $\mathrm{al}^{28}$. Heavy microbial burden $\left(1,112-5,198 \mathrm{CFU} / 100 \mathrm{~cm}^{2}\right)$ was also reported by Schmidt et al ${ }^{29}$. on plastic bed rails even after cleaning. The findings of Wirtanen et $\mathrm{al}^{30}$. of high microbial burden on the OT sandals coincides with this study. Overall the findings of bacterial burden on high touched inanimate surfaces of Hospital ' $A$ ' \& ' $C$ ' higher than Hospital ' $\mathrm{B}$ ' was statistically significant. It appears that the infection control measures are in good practice in Hospital ' $\mathrm{B}$ ' which is a private monodisciplinary specialized cardiac hospital than the other two multidisciplinary private and government general hospitals.

In this study total 232 environmental swab samples (ICU \& OT) were taken, among which approximately $75 \%$ samples were found culture positive $\&$ this was close to the findings (69.6\%) to a study conducted by Rozonska et $\mathrm{a}^{31}$. In another study, $51 \%$ of the environmental samples were found positive with different bacterial species in ICU samples ${ }^{32}$.
Among the isolates of ICU, Hospital ' $A$ ' and ' $C$ ' have predominant gram negative isolates (Table 3.5) which was statistically significant. Similar findings were reported by Jadhav et $\mathrm{a}^{33}$ that gram negative bacteria constituted the dominant colonized bacteria compared with gram positive cocci among the bacteria isolated from respiratory devices (68.85\% versus $31.14 \%)$ in Maharashtra, India. On the other hand, Hospital 'B' ICU has a predominance of gram positive organisms. Similar rates $(60.7 \%$ Vs. $39.3 \%$ ) was found by Tajeddin et $\mathrm{al}^{32}$. in a hospital of Tehran. However, in OTs the scenario was reverse where gram positive isolates were detected more than gram negative isolates in all the three hospitals-71.7\% vs. $28.3 \%$ in Hospital 'A', $68.2 \%$ vs $31.8 \%$ in Hospital 'B' \& $59.4 \%$ vs. $40.6 \%$ in Hospital ' $C$ '. This finding correlate with Kiranmai ${ }^{34}$ in Telangana, India.

In OTs of three hospitals the isolated organisms were predominantly non-pathogenic (58.7-65.9\%) (Table 3.6). Among the non-pathogens (Table 3.4) $37.8 \%$ were Micrococcus spp. (normal flora) and $19.8 \%$ were Bacillus species (contaminants). The findings of this study was in agreement with the study of Kiranmai ${ }^{34}$ in Telangana, India that reported predominance of non-pathogenic organismsBacillus spp. 45\% (contaminants) and Micrococcus spp. $33 \%$ (normal flora). In this study, among the pathogens, rate of isolation was highest for Flavobacterium spp. (13.4\%) followed by Pseudomonas spp. (8.3\%) and Acinetobacter spp. (4.6\%). No MRSA was detected from OT samples in this study.

In ICUs of three hospitals the isolated organisms were predominantly pathogenic. In Hospital 'A' ICU (69.6\%) \& Hospital 'C' ICU (88.9\%) pathogenic organisms were detected (Table 3.6). On the other hand, non-pathogens were found more (67.4\%) in Hospital 'B' ICU. In terms of isolation of organisms from ICU samples, the ICU of Hospital 'B' (private monodisciplinary cardiac specialized hospital) appeared less contaminated than the other two hospitals. In ICUs among the isolated pathogens, Acinetobacter spp. was the predominant isolate $(21.2 \%)$ followed by Pseudomonas spp. (19.2\%), Klebsiella spp. (7.7\%) \& S.aureus (4.5\%). And among the non-pathogens, Micrococcus spp. was predominant (21.8\%) followed by Bacillus spp. $(11.5 \%) \&$ Coagulase negative Staphylococcus (1.3\%) (Table 3.3). This finding was in accordance with the findings of Huang et $\mathrm{a}^{35}$. in Taiwan. 
Health care workers' (HCW) hands are major sources of transmission of nosocomial pathogens ${ }^{36}$. Culture of $41.9 \%$ hand \& $79.1 \%$ nasal swab of ICU staffs yielded growth (Table 3.7). Among the hand swabs, majority were Micrococcus spp. (66.7\%) which were non-pathogens. In Hospital 'B' no pathogens were found from the hands of the staffs. In rest two hospitals, Klebsiella spp., E. coli, Enterobacter spp., Pseudomonas spp. and S. aureus were found in a range of $5-11 \%$. Waters et $\mathrm{al}^{36}$. reported that $30 \%$ of hospital employees' hands were persistently colonized by Gram negative bacilli, including Acinetobacter, Klebsiella, Enterobacter, S. marcescens, and E. coli. In a study by Khodavaisy et $\mathrm{al}^{37}$. higher rates of colonization of Klebsiella spp. (7.9\%), Enterobacter spp. (4.7\%), E. coli (3.9\%), Acinetobacter spp. (3.1\%) and Pseudomonas spp. (2.3\%) were reported among HCWs' hands. Among the nasal swabs, most of the colonization was found in Hospital ' $\mathrm{C}$ ' which includes $11.8 \%$ of MRSA, 5.9\% of Pseudomonas spp. and $23.5 \%$ of Enterobacteriaceae. The isolation rate of S. aureus (22.2-25\%) and CoNS (17.6-25\%) was almost similar among the three hospitals. In this study the rate of nasal colonization with $S$. aureus was found in agreement with $21.7 \%$ reported by Satpathi et $\mathrm{al}^{38}$. Frequency of bacterial colonization was found higher in Hospital 'C' than Hospital 'A' \& 'B'.

\section{Conclusion}

The inanimate surfaces of ICU environment revealed unacceptable microbial burden in Hospital 'A' \& ' $\mathrm{C}$ '. Nasal \& hand swabs of Hospital 'C' ICU staffs' were also found moderately heavy colonization with MDR pathogens like MRSA. Unacceptable range of coliform organisms were found in all three ICUs. The OT environment was also found grossly contaminated though here majority of organisms were found non-pathogens. Antimicrobial resistance pattern was found high up among the pathogens. Highest resistance was observed among the Acinetobacter isolates which was found in the ICU environment.

The overall mean bacterial load \& hygiene status of high risk areas (ICU \& OT) were detected better in the private monodisciplinary specialized cardiac hospital (Hospital 'B') than multidisciplinary private general hospital (Hospital 'A') \& multidisciplinary government general hospital (Hospital 'C'). It might be due to difference in implementation and practice of infection control protocol in respective hospitals. Using the results of this study, an initiative should be undertaken for establishing regular cleaning of the hospital environment to decrease the microbial burden especially in high risk areas.

\section{References}

1. Muhammad UK, Isa MA, Aliyu ZM. Distribution of potential nosocomial pathogens isolated from environments of four selected hospitals in Sokoto, North Western Nigeria. Journal of microbiology and biotechnology research. 2013;3(1):139-43.

2. Macías AE, Muñoz JM, Bruckner DA, Galván A, Rodríguez AB, Guerrero FJ, et al. Parenteral infusions bacterial contamination in a multi-institutional survey in Mexico: considerations for nosocomial mortality. American journal of infection control. 1999 Jun $1 ; 27(3): 285-90$.

3. Rezende EM, Couto BR, Starling CE, Módena CM. Prevalence of nosocomial infections in general hospitals in Belo Horizonte. Infection control \& hospital epidemiology. 1998 Nov;19(11):872-6.

4. Mayon-White RT, Ducel G, Kereselidze T, Tikomirov E. An international survey of the prevalence of hospital-acquired infection. Journal of hospital infection. 1988 Feb 1;11:43-8.

5. Western KA, John RK, Shearer LA. Hospital infection control-an international perspective. Infection control \& hospital epidemiology. 1982 Dec;3(6):453-5.

6. Grimes DA, Peterson HB, Rosenberg MJ, Fishburne Jr JI, Rochat RW, Khan AR, et al. Sterilization-attributable deaths in Bangladesh. International journal of gynecology \& obstetrics. 1982 Apr 1;20(2):149-54.

7. Shin H, Pei Z, Martinez KA, Rivera-Vinas JI, Mendez $\mathrm{K}$, Cavallin $\mathrm{H}$, et al. The first microbial environment of infants born by C-section: the operating room microbes. Microbiome. 2015 Dec;3(1):1-6.

8. Andersen BM, Rasch M, Kvist J, Tollefsen T, Lukkassen R, Sandvik L, et al. Floor cleaning: effect on bacteria and organic materials in hospital rooms. Journal of hospital infection. 2009 Jan 1;71(1):57-65.

9. Venkatesan A, Kansal S, Patel SS, Akulwar SK. The role of hand hygiene and mobile phones in transmitting hospital acquired infection. International journal of biomedical and advance research. 2015 May 30;6:435-7.

10. Verde SC, Almeida SM, Matos J, Guerreiro D, Meneses M, Faria T, et al. Microbiological assessment of indoor air quality at different hospital sites. Research in microbiology. 2015 Sep 1;166(7):557-63. 
11. Wan GH, Chung FF, Tang CS. Long-term surveillance of air quality in medical center operating rooms. American journal of infection control. 2011 May 1;39(4):302-8.

12. Al Laham NA. Prevalence of bacterial contamination in general operating theaters in selected hospitals in the Gaza Strip, Palestine. Journal of infection and public health. 2012 Feb 1;5(1):43-51.

13. Edmiston Jr CE, Seabrook GR, Cambria RA, Brown $\mathrm{KR}$, Lewis BD, Sommers JR, et al. Molecular epidemiology of microbial contamination in the operating room environment: Is there a risk for infection?. Surgery. 2005 Oct 1;138(4):573-82.

14. Weber DJ, Anderson D, Rutala WA. The role of the surface environment in healthcare-associated infections. Current opinion in infectious diseases. 2013 Aug 1;26(4):338-44.

15. Poza M, Gayoso C, Gomez MJ, Rumbo-Feal S, Tomás M, Aranda J, et al. Exploring bacterial diversity in hospital environments by GS-FLX Titanium pyrosequencing. PLOS one. 2012 Aug 29;7(8):e44105.

16. Mohiuddin MD, Haq JA, Hoq MM, Huq F. Microbiology of nosocomial infection in Tertiary Hospitals of Dhaka city and its impact. Bangladesh journal of medical microbiology. 2010;4(2):32-8.

17. Kurutkan MN, Kara O, Eraslan IH. An implementation on the social cost of hospital acquired infections. International Journal of clinical and experimental medicine. 2015;8(3):4433.

18. Otter JA, Yezli S, Salkeld JA, French GL. Evidence that contaminated surfaces contribute to the transmission of hospital pathogens and an overview of strategies to address contaminated surfaces in hospital settings. American journal of infection control. 2013 May 1;41(5):S6-11.

19. Esteves DC, Pereira VC, Souza JM, Keller R, Simões $\mathrm{RD}$, Eller LK, et al. Influence of biological fluids in bacterial viability on different hospital surfaces and fomites. American journal of infection control. 2016 Mar 1;44(3):311-4.

20. Abreu AC, Tavares RR, Borges A, Mergulhão F, Simões M. Current and emergent strategies for disinfection of hospital environments. Journal of antimicrobial chemotherapy. 2013 Dec 1;68(12):2718-32.
21. Galvin S, Dolan A, Cahill O, Daniels S, Humphreys $\mathrm{H}$. Microbial monitoring of the hospital environment: why and how? Journal of hospital infection. 2012 Nov $1 ; 82(3): 143-51$.

22. Michels HT, Wilks SA, Noyce JO, Keevil C. Copper alloys for human infectious disease control. Stainless steel. 2005;77000(55.0):27-0.

23. Oie S, Hosokawa I, Kamiya A. Contamination of room door handles by methicillin-sensitive/methicillinresistant Staphylococcus aureus. Journal of hospital infection. 2002 Jun 1;51(2):140-3.

24. Blythe D, Keenlyside D, Dawson SJ, Galloway A. Environmental contamination due to methicillinresistant Staphylococcus aureus (MRSA). Journal of hospital infection. 1998 Jan 1;38(1):67-9.

25. Dancer SJ. How do we assess hospital cleaning? A proposal for microbiological standards for surface hygiene in hospitals. Journal of hospital infection. 2004 Jan 1;56(1):10-5.

26. Bauer AW, Kirby WMM, Sherris JC, Turck M. Antibiotic susceptibility testing by a standardized single disk method. American journal of clinical pathology. 1966;4(45):493-496.

27. Shams AM, Rose LJ, Edwards JR, Cali S, Harris AD, Jacob JT, et al. Assessment of the overall and multidrugresistant organism bioburden on environmental surfaces in healthcare facilities. Infection control and hospital epidemiology. 2016 Dec;37(12):1426.

28. Dancer SJ, White L, Robertson C. Monitoring environmental cleanliness on two surgical wards. International journal of environmental health research. 2008 Oct 1;18(5):357-64.

29. Schmidt MG, Anderson T, Attaway III HH, Fairey S, Kennedy C, Salgado CD. Patient environment microbial burden reduction: a pilot study comparison of 2 terminal cleaning methods. American journal of infection control. 2012 Aug 1;40(6):559-61.

30. Wirtanen G, Nurmi S, Kalliohaka T, Mattila I, Heinonen K, Enbom S, et al. Surface and air cleanliness in operating theatre environments. Europian journal of parenteral and pharmaceutical sciences. 2012;17(3):1-7.

31. Różańska A, Romaniszyn D, Chmielarczyk A, Bulanda M. Bacteria contamination of touch surfaces in Polish hospital wards. Medycyna pracy. 2017 Jul 1;68(4):459. 
32. Tajeddin E, Rashidan M, Razaghi M, Javadi SS, Sherafat $\mathrm{SJ}$, Alebouyeh M, et al. The role of the intensive care unit environment and health-care workers in the transmission of bacteria associated with hospital acquired infections. Journal of infection and public health. 2016 Jan 1;9(1):13-23.

33. Jadhav S, Sahasrabudhe T, Kalley V, Gandham N. The microbial colonization profile of respiratory devices and the significance of the role of disinfection: a blinded study. Journal of clinical and diagnostic research: JCDR. 2013 Jun;7(6):1021.

34. Kiranmai S, Madhavi K. Microbiological surveillance of operation theatres, intensive care units and labor room of a teaching hospital in Telangana, India. International journal of research in medical sciences. 2016 Dec;4(12):5256.

35. Huang PY, Shi ZY, Chen CH, Den W, Huang HM, Tsai JJ. Airborne and surface-bound microbial contamination in two intensive care units of a medical center in central Taiwan. Aerosol and air quality research. 2012;13(3):1060-9.
36. Waters V, Larson E, Wu F, San Gabriel P, Haas J, Cimiotti J, et al. Molecular epidemiology of gram-negative bacilli from infected neonates and health care workers' hands in neonatal intensive care units. Clinical infectious diseases. 2004 Jun 15;38(12):1682-7.

37. Khodavaisy S, Nabili M, Davari B, Vahedi M. Evaluation of bacterial and fungal contamination in the health care workers' hands and rings in the intensive care unit. Journal of preventive medicine and hygiene. 2011 Dec 1;52(4):215-8.

38. Satpathi PS, Maity AB, Mukherjee P, Satpathi S. Nasal carriage of staphylococcus aureus and the quantum of their methicillin resistance amongst the health care workers in a peripheral tertiary care centre of Eastern India. Journal of evolution of medical and dental sciences. 2015 Nov 9;4(90):15537-43. 